\title{
The prevalence change of hyperlipidemia and hyperglycemia and the effectiveness of yearly physical examinations: an eight-year study in Southwest China
}

Wei Gan, Ying Liu, Kai-Hong Luo, Shan-Shan Liang, Hui Wang, Meng Li, Yan-Xing Zhang and Heng-Jian Huang*

\begin{abstract}
Background: The aim of this study was to investigate the prevalence changes of hyperlipidemia and hyperglycemia from 2009 to 2016 and the effectiveness of yearly physical examinations to hyperlipidemia and hyperglycemia prevention in Chengdu.

Methods: A total of 794 residents (499 males) who have undergone annual health check-ups for 8 consecutive years (from 2009 to 2016) in Chengdu, a city in southwest China were selected as the follow-up group, 7226 residents in 2009 and 75,068 residents in 2016 who underwent health examinations in the same hospital were chosen to be the contemporary control group. The concentration of fasting serum triglyceride(TG), total cholesterol(TC), low density lipoprotein cholesterol( $(\mathrm{DL}-\mathrm{C})$, high density lipoprotein cholesterol $(\mathrm{HDL}-\mathrm{C})$ and glucose were measured and compared among these groups.

Results: There was a clear rise in the prevalence of hypercholesterolemia and hyperglycemia from 2009 to 2016 ( $p<0$. 05). The follow-up group didn't show difference in levels of serum lipids and glucose compared with the general population after an 8-years' consecutive physical examination $(p>0.05)$, the follow-up cohort in the 8th year exhibited significant increases in serum total cholesterol and glucose compared with the 1st year $(p<0.05)$.

Conclusion: The prevalence of hypercholesterolemia and hyperglycemia were increased significantly from 2009 to 2016. Annual physical examination didn't show a positive effect in the prevention of hypercholesterolemia and hyperglycemia. Health education should be improved to ensure the fulfillment of the preventive objective of yearly physical examination.
\end{abstract}

Keywords: Annual physical examination, Serum lipids, Serum glucose, Hypercholesterolemia, Hyperglycemia

\section{Background}

Coronary heart disease (CHD) is the leading cause of death both in worldwide and China [1,2]. In 2015, cardiovascular diseases (CVD) became the first leading cause of death for Chinese, $45.01 \%$ deaths in rural area and $42.61 \%$ deaths in urban area were caused by CVD [3].

Hyperlipidemia, especially elevated levels of lowdensity lipoprotein (LDL) cholesterol, is an independent risk factor for cardiovascular diseases [4]. The China Health and Nutrition Survey in 2012 [5] revealed that

\footnotetext{
* Correspondence: huanghenjian@sina.com

Department of Laboratory Medicine, West China Hospital, Sichuan University, No. 37 Guoxue Alley, Wuhou district Chengdu, Sichuan 610041, China
}

the prevalence of total cholesterol $(\mathrm{TC}) \geq 6.22 \mathrm{mmol} / \mathrm{L}$ among males and females aged over 18 were 4.7 and 5 . $1 \%$, respectively, and the prevalence rates of triglyceride (TG) $\geq 2.26 \mathrm{mmol} / \mathrm{L}$ among males and females were 16.7 and $9.8 \%$, respectively.

Diabetes is also a major risk factor for cardiovascular disease, the prevalence of diabetes is increasing constantly in China. The overall prevalence of diabetes was estimated to be $11.6 \%$ in the Chinese adult population, with $12.1 \%$ among men and $11.0 \%$ among women [6].

Early detection of dyslipidemia and hyperglycemia is an important strategy for early prevention and treatment. Nowadays, yearly health examination, including 
serum lipid and glucose screening has become a welfare provided by many employers. Despite no evidence in favour, such kind of periodic physical examination is considered an important and effective measure in the prevention of illness, and is very popular national-wide.

This study aimed to answer the scientific question "How much do yearly health examinations do to the control of hyperlipidemia and hyperglycemia". Moreover, this study also investigated the changes in the prevalence and incidence rates of hyperlipidemia and hyperglycemia from 2009 to 2016; and explored the relationship between age/sex and serum lipids and glucose.

\section{Methods}

\section{Participants}

All the participants were recruited from the physical examination center, West China Hospital, Sichuan University. A total of 794 local residents who started with a yearly health examination in 2009 for 8 consecutive years (from 2009 to 2016) were selected as the follow-up group, which was used to investigate the effectiveness of yearly physical examination to the control of hyperlipidemia and hyperglycemia. All the residents who went for health checkups in 2009 and 2016 were selected to be the contemporary control group and used to investigate the prevalence changes of hyperlipidemia and hyperglycemia from 2009 to 2016. All the participants were subdivided into 10-year age classes ( $\leq 30$ years, $31-40$ years, $41-50$ years, $51-60$ years, $61-70$ years, $\geq 71$ years). All the participants were from urban area of Chengdu city, Sichuan province of China. They were all common staffs of local company or agency, no special records about their life style were found in the subjects.

\section{Serum lipids and glucose measurement}

Serum TG, TC, LDL-C, HDL-C and serum glucose levels were measured after at least a 10-h overnight fasting among all study participants, the measurements were performed on a Cobas8000 system (Roche Diagnostics $\mathrm{GmbH}$, Germany) in the Department of Laboratory Medicine, West China Hospital, Sichuan University, which is a College of American Pathologists (CAP)accredited laboratory.

\section{Diagnostic criteria}

According to the guidelines on prevention and treatment of blood lipid abnormality in Chinese adults [7], TC $\geq 6$. $2 \mathrm{mmol} / \mathrm{L}$ was defined as hypercholesterolemia and $\mathrm{TG} \geq 2.3 \mathrm{mmol} / \mathrm{L}$ was diagnosed as hypertriglyceridemia. T2DM was identified according to the criteria of Chinese Diabetes Society [8], fasting serum glucose $\geq 7$. $0 \mathrm{mmol} / \mathrm{L}$ was defined as hyperglycemia.

\section{Strategies for evaluating the effectiveness of yearly physical examination to hyperlipidemia and hyperglycemia control}

Three strategies were used to evaluate the effectiveness of consecutive physical examination. 1) A cross-sectional comparison: Compare the levels of TG, TC and glucose between the 8th year of follow-up group and 2016 of control group. 2) A longitudinal comparison: Compare the levels of TG, TC and glucose between the 1st and the 8th physical examination year in the follow-up group. 3) Calculate the numbers of participants who had a bad hyperlipidemia or hyperglycemia control during the 8 years in the follow-up group. A "bad hyperlipidemia or hyperglycemia control" is defined as persons with fasting serum triglyceride $\geq 2.3 \mathrm{mmol} / \mathrm{L}$, or cholesterol $\geq 6.2 \mathrm{mmol} / \mathrm{L}$, or blood glucose $\geq 7.0 \mathrm{mmol} / \mathrm{L}$ for $\geq 3$ times among the total 8 examinations.

\section{Statistical analysis}

All statistical analyses were performed using the SPSS 16.0 software program (SPSS Inc. USA). Serum lipids and glucose concentration distributed normally and were expressed as the mean \pm standard deviation. Differences between gender groups were assessed by independent t-test. Differences among age groups were tested by one-way ANOVA, followed by S-N-K test for equal variances assumed and Dunnett's T3 test for equal variances not assumed. All $p$ values were two-sided, and a $p$ value $<0.05$ was considered statistically significant.

\section{Results}

The relationship between age/sex and serum lipids and glucose

The number of the participants in each age group were listed in Table 1, the year of age spanned from 12 to 98 . Data in 2016 were used to investigate the relationship between age/sex and these measured variables.

The change patterns of serum lipids and glucose with aging were different from each other (Table 2). In detail, TG in female exhibited the most obvious age-dependent increase, the level of TG in $\leq 30$ year group was $0.88 \pm 0$. $44 \mathrm{mmol} / \mathrm{L}$, a 1.76 -fold increase was found in $\geq 71$ year group $(1.55 \pm 0.73 \mathrm{mmol} / \mathrm{L})(p<0.001)$. Glucose in both gender also exhibited a demonstrated age-dependent increase, a 1.25- and 1.26-fold higher level for male and female were present in $\geq 71$ year group compared with the $\leq 30$ year group (both $p<0.001$ ), respectively. HDL-C rose slightly during all the life-span, for male, a 1.12-fold increase were found in the $\geq 71$ year group compared with the $\leq 30$ year group $(p<0.001)$, for female, even more slightly changes were present from the young to the old. TG in male, TC and LDL-C in both gender followed the pattern of "first increase, and then decrease slightly". However, the levels of the three variables in 
Table 1 Numbers of participants in each age group

\begin{tabular}{|c|c|c|c|c|c|c|c|c|c|c|c|c|}
\hline \multirow[b]{2}{*}{ Age group } & \multicolumn{2}{|l|}{2009} & \multirow[t]{2}{*}{ Total } & \multicolumn{2}{|l|}{2016} & \multirow[t]{2}{*}{ Total } & \multicolumn{2}{|c|}{ 1st year of annual checkup } & \multirow[t]{2}{*}{ Total } & \multicolumn{2}{|c|}{ 8th year of annual checkup } & \multirow[t]{2}{*}{ Tota } \\
\hline & Male & Female & & Male & Female & & Male & Female & & Male & Female & \\
\hline$\leq 30 y$ & 196 & 185 & & 7268 & 8136 & & 42 & 33 & & 3 & 5 & \\
\hline $31-40 y$ & 860 & 769 & & 10,976 & 8986 & & 111 & 80 & & 60 & 47 & \\
\hline $41-50 y$ & 1185 & 1016 & & 10,557 & 8478 & & 153 & 99 & & 137 & 109 & \\
\hline $51-60 y$ & 778 & 798 & & 6772 & 4635 & & 126 & 50 & & 156 & 85 & \\
\hline $61-70 y$ & 362 & 417 & & 2609 & 2075 & & 47 & 26 & & 80 & 43 & \\
\hline$\geq 71 y$ & 316 & 344 & & 2699 & 1877 & & 20 & 7 & & 50 & 19 & \\
\hline Total & 3697 & 3529 & 7226 & 40,881 & 34,187 & 75,068 & 499 & 295 & 794 & 486 & 308 & 794 \\
\hline
\end{tabular}

$\geq 71$ year group were still higher than $\leq 30$ year group. The turning points from "increase to decrease" were at the age of 50, 60 and 60 for TG in male, TC and LDL-C in both genders, respectively.

Under the age of menopause, males always exhibited higher levels of serum lipids and glucose compared with age-matched female $(p<0.001)$ except for HDL-C. However, females experienced their highest increase during the menopausal period, and decreased much more slightly than male over the age of the "turning point". As a result, female showed higher levels of serum lipids and glucose than male in the latter age stages.

\section{The changes in the prevalence of hyperlipidemia and hyperglycemia from 2009 to 2016}

According to the diagnostic criteria, The incidence rates of hypertriglyceridemia, hypercholesterolemia and hyperglycemia in 2009 and 2016 were listed in Table 3. Since the number of participants in $\leq 30$ year group and $\geq 71$ year group in 2009 were small (see Table 1), to avoid introducing bias in calculating the incidence rate, we merged $\leq 30$ year group and 31-40 year group to be $\leq 40$ year group, $61-70$ year group and $\geq 71$ year group to be $\geq 61$ year group.

Concerning the year of 2016, the highest incidence rate of hypertriglyceridemia in male was $24.9 \%$ in $41-50$ year group, $13.2 \%$ in $\geq 61$ year female group. The maximum incidence rate of hypercholesterolemia in male was $10.4 \%$ in
51-60 year group, $18.1 \%$ in $\geq 61$ female year group. As for hyperglycemia, participants in $\geq 61$ year group exhibited the highest prevalence rate, which was 17.5 and $12.8 \%$ in male and female, respectively.

The year of 2016 witnessed great changes in the lipids profile and glucose in the past 8 years. The incidence rate of hypertriglyceridemia decreased in all the age groups except for the $\leq 40 \mathrm{y}$ female group. A significant increase in hypercholesterolemia prevalence was seen in all the age groups, the highest increase rate was 3.5 folds in 4150 year group in female. The incidence rate of hyperglycemia also showed an obvious increase from 2009 to 2016 in all the age group except for $\leq 40$ year in male. A 1.7 -fold increase was seen in $\geq 61$ year male group.

\section{Effectiveness of yearly physical examination to hyperlipidemia and hyperglycemia control}

By using the 3 listed strategies, we got the following results (Table 4). There were no significant differences in TG, TC and glucose between the 2016 control group and the 8th year follow-up group (all $p>0.05$ ). For the follow-up group, TC and glucose increased significantly from the 1st to the 8 th year, while TG showed a slightly decrease (Table 4). Of the total 794 participants, 160 had a bad hypertriglyceridemia control, 36 had a bad hypercholesterolemia control, and 68 had a bad hyperglycemia control.

Table 2 The relationship between age/sex and serum lipids and glucose

\begin{tabular}{|c|c|c|c|c|c|c|c|c|c|c|}
\hline \multirow[b]{2}{*}{ Age group } & \multicolumn{2}{|l|}{$\mathrm{TG}$} & \multicolumn{2}{|l|}{$\mathrm{TC}$} & \multicolumn{2}{|l|}{ LDL-C } & \multicolumn{2}{|l|}{$\mathrm{HDL}-\mathrm{C}$} & \multicolumn{2}{|l|}{ Glucose } \\
\hline & Male & Female & Male & Female & Male & Female & Male & Female & Male & Female \\
\hline$\leq 30 y$ & $1.42 \pm 0.84$ & $0.88 \pm 0.44^{\#}$ & $4.58 \pm 0.83$ & $4.38 \pm 0.73^{\#}$ & $2.57 \pm 0.67$ & $2.20 \pm 0.57^{\#}$ & $1.38 \pm 0.32$ & $1.78 \pm 0.38^{\#}$ & $4.96 \pm 0.63$ & $4.86 \pm 0.44^{\#}$ \\
\hline $31-40 y$ & $1.69 \pm 0.94^{*}$ & $1.00 \pm 0.52^{*, \#}$ & $4.83 \pm 0.84^{*}$ & $4.54 \pm 0.78^{*}, \#$ & $2.74 \pm 0.67^{*}$ & $2.35 \pm 0.62^{*, \#}$ & $1.34 \pm 0.33^{*}$ & $1.74 \pm 0.39^{*}, \#$ & $5.14 \pm 0.83^{*}$ & $5.00 \pm 0.55^{*, \#}$ \\
\hline $41-50 y$ & $1.85 \pm 0.99^{*}$ & $1.17 \pm 0.62^{* \text {,\# }}$ & $5.04 \pm 0.88^{*}$ & $4.90 \pm 0.83^{*, \#}$ & $2.87 \pm 0.72^{*}$ & $2.62 \pm 0.67^{* \text {,\# }}$ & $1.36 \pm 0.34^{*}$ & $1.76 \pm 0.41^{* \text {,\# }}$ & $5.42 \pm 1.26^{*}$ & $5.14 \pm 0.66^{*}$ \\
\hline J1-0uy & $1.78 \pm 0.94^{*}$ & $1.45 \pm 0.82^{*, \#}$ & $5.05 \pm 0.91^{*}$ & $5.40 \pm 0.91^{*, \#}$ & $2.88 \pm 0.73^{*}$ & $2.98 \pm 0.74^{*, \#}$ & $1.38 \pm 0.36$ & $1.77 \pm 0.44^{\#}$ & $5.79 \pm 1.68^{*}$ & $5.42 \pm 1.00^{*}$, \# \\
\hline $61-70 y$ & $1.55 \pm 0.78^{*}$ & $1.56 \pm 0.78^{*}$ & $4.99 \pm 0.95^{*}$ & $5.37 \pm 0.95^{*}$,\# & $2.82 \pm 0.76^{*}$ & $2.95 \pm 0.77^{*}$,\# & $1.46 \pm 0.38^{*}$ & $1.74 \pm 0.45^{*}$,\# & $6.06 \pm 1.73^{*}$ & $5.82 \pm 1.44^{*}$, \# \\
\hline$\geq 70 y$ & $1.38 \pm 0.67^{*}$ & $1.55 \pm 0.74^{*}, \#$ & $4.87 \pm 0.96^{*}$ & $5.30 \pm 1.06^{*, \#}$ & $2.69 \pm 0.77^{*}$ & $2.84 \pm 0.85^{*, \#}$ & $1.54 \pm 0.44^{*}$ & $1.79 \pm 0.49^{\#}$ & $6.20 \pm 1.68^{*}$ & $6.11 \pm 1.63^{*,+}$ \\
\hline
\end{tabular}

* $p<0.001$ compared with sex-matched $\leq 30$ year group

\# $p<0.001$ compared with age-matched male 
Table 3 Percentages of hypertriglyceridemia, hypercholesterolemia and hyperglycemia in 2009 and 2016

\begin{tabular}{|c|c|c|c|c|c|c|c|c|c|c|c|c|}
\hline \multirow{3}{*}{ Age group } & \multicolumn{4}{|c|}{$\%$ with $\mathrm{TG} \geq 2.3 \mathrm{mmol} / \mathrm{L}$} & \multicolumn{4}{|c|}{$\%$ with TC $\geq 6.2 \mathrm{mmol} / \mathrm{L}$} & \multicolumn{4}{|c|}{$\%$ with Glucose $\geq 7.0 \mathrm{mmol} / \mathrm{L}$} \\
\hline & \multicolumn{2}{|l|}{ Male } & \multicolumn{2}{|c|}{ Female } & \multicolumn{2}{|l|}{ Male } & \multicolumn{2}{|c|}{ Female } & \multicolumn{2}{|l|}{ Male } & \multicolumn{2}{|c|}{ Female } \\
\hline & 2009 & 2016 & 2009 & 2016 & 2009 & 2016 & 2009 & 2016 & 2009 & 2016 & 2009 & 2016 \\
\hline$\leq 40 y$ & 26.5 & 17.0 & 1.3 & 2.1 & 3.8 & 5.3 & 1.0 & 2.2 & 2.1 & 1.1 & 0.0 & 0.2 \\
\hline $41-50 y$ & 30.1 & 24.9 & 7.0 & 5.4 & 4.2 & 9.5 & 1.9 & 6.7 & 4.7 & 5.3 & 1.1 & 1.3 \\
\hline $51-60 y$ & 23.8 & 22.5 & 13.4 & 11.5 & 4.2 & 10.4 & 9.8 & 17.9 & 7.3 & 11.0 & 4.3 & 4.0 \\
\hline$\geq 61 y$ & 15.0 & 11.9 & 20.6 & 13.2 & 6.3 & 8.9 & 15.2 & 18.1 & 10.2 & 17.5 & 10.1 & 12.8 \\
\hline
\end{tabular}

\section{Discussion}

Periodic physical examination is indeed very important in some situations, such as cancer screening [9]. However, its utility in the control of hyperlipidemia and hyperglycemia has not been fully elucidated. In this study, we firstly investigated the relationship between age/sex and serum lipids and glucose, it was demonstrated that TG in male followed the pattern of first increase, then decrease. TG in female showed an obvious increase with aging. TC and LDL-C in both genders followed the pattern of increase, decrease. HDL-C and glucose in both genders followed an age-dependent increasing manner. Which is similar to the report of Loh [10] and Harman [11]. These different lipids and glucose profiles in different age/sex persons have shown a need for age/sex dependent hyperlipidemia and hyperglycemia diagnostic cutoff value. Moreover, a clear "menopause phenomenon" which referred to that females showed a clear rise in the levels of lipids and glucose than males during the menopausal period was seen in all the groups, this phenomenon might be attributed to estrogen withdrawal in the menopausal period and indicated the need for special attention for menopausal women.

The levels of total cholesterol, LDL-C and glucose increased significantly during the 8 years, which is in accordance with the report $[5,6,12-15]$, and partially in agreement with Kheirandish $M$ [16] who showed a favorable trends in TC, triglycerides, HDL-C, but increased trends in glucose level in a 10 years follow-up. Our results showed an urgent need for population-based education on health eating and physical activity. In contrast with the reported data, TG decreased from 2009 to 2016 in our study. Firstly, we rechecked the history records of "proficiency test" which was organized by College of American Pathologists (CAP) and didn't find any bad history records. After excluding the measurement error, we noticed that the physical examination center has delivered an extensive pre-examination education to the participants through various ways in recent years, therefore, we concluded that participants were in a more strictly fasting state in 2016 compared with 2009, which could give a lower TG results.

We evaluated the effectiveness of yearly physical examination to hyperlipidemia and hyperglycemia control through 3 aspects (see Methods), the results showed that after 8 years' consecutive physical examination, the cohort were not "healthier" than the general population. Compared with the 1st year, the cohort in the 8th year showed significant increases in serum total cholesterol and glucose. Moreover, some participants showed a bad hyperlipidemia and hyperglycemia control. Based on these results, we demonstrated that effectiveness of periodic physical examination to the control of hyperlipidemia and hyperglycemia was limited. Very few researches focused on the same topic as we did. Lau et al. [17] showed a large population-based multifactorial screening on 10-year

Table 4 Levels of TG, TC and glucose in 2016 of the control group, the 1st and 8th physical examination year of the follow-up group

\begin{tabular}{|c|c|c|c|c|c|c|c|c|c|}
\hline & & \multicolumn{2}{|l|}{$\leq 40 y$} & \multicolumn{2}{|l|}{$41-50 y$} & \multicolumn{2}{|l|}{$51-60 y$} & \multicolumn{2}{|l|}{$\geq 61 y$} \\
\hline & & Male & Female & Male & Female & Male & Female & Male & Female \\
\hline \multirow[t]{3}{*}{$\overline{\mathrm{TG}}$} & 2016 & $1.59 \pm 0.94$ & $0.88 \pm 0.43$ & $1.85 \pm 0.99$ & $1.17 \pm 0.62$ & $1.78 \pm 0.94$ & $1.45 \pm 0.75$ & $1.46 \pm 0.73$ & $1.55 \pm 0.76$ \\
\hline & 1st year & $1.81 \pm 1.25$ & $0.95 \pm 0.48$ & $2.15 \pm 1.44$ & $1.10 \pm 0.61$ & $1.87 \pm 0.99$ & $1.45 \pm 0.89$ & $1.74 \pm 0.83$ & $1.87 \pm 1.15$ \\
\hline & 8th year & $1.73 \pm 1.15$ & $0.98 \pm 0.45$ & $1.75 \pm 0.91^{*}$ & $1.10 \pm 0.55$ & $1.70 \pm 0.84$ & $1.75 \pm 0.78$ & $1.53 \pm 0.79^{*}$ & $1.65 \pm 0.83$ \\
\hline \multirow[t]{3}{*}{$\mathrm{TC}$} & 2016 & $4.73 \pm 0.84$ & $4.37 \pm 0.72$ & $5.04 \pm 0.88$ & $4.90 \pm 0.83$ & $5.05 \pm 0.91$ & $5.40 \pm 0.91$ & $4.93 \pm 0.95$ & $5.33 \pm 1.00$ \\
\hline & 1st year & $4.34 \pm 0.72$ & $4.20 \pm 0.89$ & $4.81 \pm 0.87$ & $4.39 \pm 0.67$ & $4.69 \pm 0.83$ & $4.91 \pm 0.85$ & $4.84 \pm 0.72$ & $5.23 \pm 0.93$ \\
\hline & 8th year & $4.77 \pm 0.78^{*}$ & $4.61 \pm 0.75^{*}$ & $4.94 \pm 0.87$ & $4.90 \pm 0.80^{*}$ & $5.05 \pm 0.87^{*}$ & $5.22 \pm 1.02^{*}$ & $4.95 \pm 0.93$ & $5.29 \pm 1.01$ \\
\hline \multirow[t]{3}{*}{ Glucose } & 2016 & $5.06 \pm 0.76$ & $4.86 \pm 0.44$ & $5.42 \pm 1.26$ & $5.14 \pm 0.66$ & $5.79 \pm 1.68$ & $5.42 \pm 1.00$ & $6.13 \pm 1.71$ & $5.96 \pm 1.54$ \\
\hline & 1st year & $4.53 \pm 0.41$ & $4.49 \pm 0.38$ & $4.83 \pm 0.86$ & $4.65 \pm 0.46$ & $4.91 \pm 1.00$ & $4.89 \pm 0.75$ & $5.00 \pm 0.84$ & $4.96 \pm 0.78$ \\
\hline & 8th year & $5.14 \pm 0.45^{*}$ & $5.03 \pm 0.45^{*}$ & $5.33 \pm 0.78^{*}$ & $5.10 \pm 0.51^{*}$ & $5.55 \pm 1.31^{*}$ & $5.38 \pm 0.77^{*}$ & $5.88 \pm 1.44^{*}$ & $5.69 \pm 0.93^{*}$ \\
\hline
\end{tabular}

* $: p<0.05$ compared with age-matched group of the 1 st physical examination year 
incidence of diabetes did not have a preventive effect on diabetes at population level.

As it is known, early stage of hyperlipidemia and hyperglycemia do not cause any discomfort to the body, but once be diagnosed, one needs to start treatment of therapeutic lifestyle change (TLC), including decline in energy intake and increase in physical activity. Lack of consistent health education and general practitioner in China makes these sub-healthy people difficult to insist on TLC treatment. The awareness, treatment, and control rate of dyslipidemia were low among adults in China [18]. Several researches have emphasized the importance of good lifestyle. CHOI et al. [19] have shown that the intervention group (who had consistent diet consultation) had a much better serum lipids control than the control group. Lu et al. [20] found that nursing interventions in public health centers demonstrated the effect of significantly reducing the values of blood pressures and blood sugars of the elderly in communities. However, a lack of lifestyle recommendations and use of preventive medication is a common problem. A study in Australia found that the vast majority of individuals at risk of or with CVD did not achieve preventive recommendations [21].

The limitation of this study is that the medication information of the participants were not included, which may influence the results of the relationship between serum lipids and glucose and age/sex (Table 2).

\section{Conclusions}

Increasing age and estrogen withdrawal in perimenopause are dangerous factors for hyperlipidemia and hyperglycemia. The prevalence of hypercholesterolemia and hyperglycemia were increased significantly from 2009 to 2016. Annual physical examination didn't show a positive effect in the prevention of hypercholesterolemia and hyperglycemia. We have well-established systems to find out the health problems, but more attention should be paid on the improvement of health education and popularization of general practitioners to ensure the fulfillment of the preventive objective of yearly physical examination.

\section{Abbreviations}

Glu: Glucose; HDL-C: High density lipoprotein-cholesterol; LDL-C: Low density lipoprotein -cholesterol; TC: Total cholesterol; TG: Triglyceride

\section{Acknowledgements}

We thank Zi-Bing Deng and Fei Chen for their valuable suggestion on the statistical analysis.

\section{Funding}

This study was supported by the National Natural Science Foundation of China (81401716) to Wei Gan. The funding source had no role in design, in the collection, analysis, and interpretation of data; in the writing of the manuscript; and in the decision to submit the manuscript for publication.

\section{Availability of data and materials}

The datasets generated and analyzed during the current study can be obtained from the laboratory information system (LIS) of the Department of Laboratory Medicine, West China Hospital, Sichuan University, and are also available from the corresponding author on reasonable request.

\section{Authors' contributions}

Designed the experiments: WG, HJH. Analyzed the data: YL. Wrote the paper: WG, YL, HJH. Involved in acquisition of analysis data files from cohort database: KHL, SSL, HW, ML, and YXZ. Contributed to interpretation of results: WG, YL, HJH. Critically reviewed drafts of the manuscript and made comments to improve clarity: WG, YL, KHL, SSL, HW, ML, YXZ, and HJH. All authors read and approved the final manuscript.

\section{Ethics approval and consent to participate}

The study was approved by the Institutional Ethics Committee of West China Hospital of Sichuan University and complied with Declaration of Helsinki. Written informed consent was obtained from all subjects.

\section{Consent for publication}

Not applicable.

\section{Competing interests}

The authors declare that they have no competing interests.

\section{Publisher's Note}

Springer Nature remains neutral with regard to jurisdictional claims in published maps and institutional affiliations.

Received: 22 November 2017 Accepted: 25 March 2018

Published online: 04 April 2018

\section{References}

1. Zhou M, Wang H, Zhu J, Chen W, Wang L, Liu S, Li Y, Wang L, Liu Y, Yin P, et al. Cause-specific mortality for 240 causes in China during 1990-2013: a systematic subnational analysis for the global burden of disease study 2013. Lancet. 2016:387:251-72.

2. Collaborators GBDCoD. Global, regional, and national age-sex specific mortality for 264 causes of death, 1980-2016: a systematic analysis for the global burden of disease study 2016. Lancet. 2017;390:1151-210.

3. Chen W, Gao R, Liu L, Zhu M, Wang W, Wang Y, Wu Z, Li H, Zheng Z, Jiang L, Hu S. Outline of the report on cardiovascular diseases in China, 2016. Chinese Circ J. 2016;32:10.

4. Kannel WB, Castelli WP, Gordon T, McNamara PM. Serum cholesterol, lipoproteins, and the risk of coronary heart disease. The Framingham study. Ann Intern Med. 1971;74:1-12.

5. National Health and Family Planning Commission, NHFPC. Report on Chinese resident's chronic disease and nutrition (2015), vol. 11. Beijing: People's Medical Publishing House; 2015. p. 18.

6. Xu Y, Wang L, He J, Bi Y, Li M, Wang T, Wang L, Jiang Y, Dai M, Lu J, et al. Prevalence and control of diabetes in Chinese adults. JAMA. 2013;310:948-59.

7. Joint Committee for Developing Chinese guidelines on Prevention and Treatment of Dyslipidemia in Adults. Chinese guidelines on prevention and treatment of dyslipidemia in adults, 2016 revised edition. Chinese Circulation J. 2016;31:17

8. Chinese Diabetes Society. Guidelines for prevention and control of type 2 diabetes in China (2013). Chinese J Diabetes. 2014;88:64.

9. Fenton JJ, Cai Y, Weiss NS, Elmore JG, Pardee RE, Reid RJ, Baldwin LM. Delivery of cancer screening: how important is the preventive health examination? Arch Intern Med. 2007;167:580-5.

10. Loh TP, Ma S, Heng D, Khoo CM. Age-related changes in the Cardiometabolic profiles in Singapore resident adult population: findings from the National Health Survey 2010. PLoS One. 2016;11:e0162102.

11. Harman $J L$, Griswold ME, Jeffries NO, Sumner AE, Sarpong DF, Akylbekova EL, Walker ER, Wyatt SB, Taylor HA Jr. Age is positively associated with highdensity lipoprotein cholesterol among African Americans in cross-sectional analysis: the Jackson heart study. J Clin Lipidol. 2011;5:173-8.

12. Bakx JC, van den Hoogen HJ, Deurenberg $P$, van Doremalen J, van den Bosch WJ. Changes in serum total cholesterol levels over 18 years in a cohort of men and women: the Nijmegen cohort study. Prev Med. 2000;30: $138-45$. 
13. Li JZ, Wang S, Dong J, Zhu LH, Li YL, Gao DL, Tian YP, Dong L, Yang L, Li SK. Present status of serum lipid levels in Beijing professional populations and its trend of changes over 15 years-a collaborative study of seven research and clinical laboratories in Beijing. Clin Chim Acta. 2005;352:199-207.

14. Gupta R, Guptha S, Agrawal A, Kaul V, Gaur K, Gupta VP. Secular trends in cholesterol lipoproteins and triglycerides and prevalence of dyslipidemias in an urban Indian population. Lipids Health Dis. 2008;7:40.

15. Arai H, Yamamoto A, Matsuzawa Y, Saito Y, Yamada N, Oikawa S, Mabuchi H, Teramoto T, Sasaki J, Nakaya N, et al. Serum lipid survey and its recent trend in the general Japanese population in 2000. J Atheroscler Thromb. 2005:12:98-106

16. Kheirandish M, Asgari S, Lotfaliany M, Bozorgmanesh M, Saadat N, Tohidi M, Azizi F, Hadaegh F. Secular trends in serum lipid levels of a middle eastern adult population; 10 years follow up in Tehran lipid and glucose study. Lipids Health Dis. 2014;13:20.

17. Lau CJ, Pisinger C, Husemoen LLN, Jacobsen RK, Linneberg A, Jorgensen T, Glumer C. Effect of general health screening and lifestyle counselling on incidence of diabetes in general population: Inter99 randomised trial. Prev Med. 2016;91:172-9.

18. Pan L, Yang Z, Wu Y, Yin RX, Liao Y, Wang J, Gao B, Zhang L, China National Survey of chronic kidney disease working $G$. The prevalence, awareness, treatment and control of dyslipidemia among adults in China. Atherosclerosis. 2016;248:2-9.

19. Choi YY, Kim KY. Effects of physical examination and diet consultation on serum cholesterol and health-behavior in the Korean pilots employed in commercial airline. Ind Health. 2013;51:603-11.

20. Lu KY, Lin PL, Tzeng LC, Huang KY, Chang LC. Effectiveness of case management for community elderly with hypertension, diabetes mellitus, and hypercholesterolemia in Taiwan: a record review. Int J Nurs Stud. 2006; 43:1001-10.

21. Gonzalez-Chica DA, Dal Grande E, Bowden J, Musker M, Hay P, Stocks N. Are we reducing the risk of cardiovascular disease and improving the quality of life through preventive health care? Results of a population-based study in South Australia. Prev Med. 2017;99:164-70.

\section{Submit your next manuscript to BioMed Central and we will help you at every step:}

- We accept pre-submission inquiries

- Our selector tool helps you to find the most relevant journal

- We provide round the clock customer support

- Convenient online submission

- Thorough peer review

- Inclusion in PubMed and all major indexing services

- Maximum visibility for your research

Submit your manuscript at www.biomedcentral.com/submit

) Biomed Central 